Revue générale

\title{
Place de l'AMP-activated protein kinase dans le préconditionnement ischémique rénal
}

\author{
Role of AMP-activated protein kinase in renal ischemic preconditioning \\ Pauline Erpicum ${ }^{a}$, Jean-Marie Krzesinski ${ }^{a, b}$, François Jouret ${ }^{\mathrm{a}, *, \mathrm{~b}}$ \\ a Service de néphrologie, université de Liège CHU (ULg CHU), CHU de Liège, tour 1, $6^{e}$ étage, avenue de l'Hôpital, 1 B-4000 Liège, Belgique \\ ${ }^{\mathrm{b}}$ GIGA Cardiovascular Sciences, université de Liège, tour 3, $5^{e}$ étage, avenue de l'Hôpital, 1 B-4000 Liège, Belgique
}

\section{N F O A R T I C L E}

\section{Historique de l'article :}

Reçu le 11 janvier 2013

Accepté le 15 octobre 2013

\section{Mots clés :}

Préconditionnement ischémique

AMP-activated protein kinase

Transplantation rénale

Metformine

\begin{abstract}
R É S U M É
La transplantation rénale représente aujourd'hui le traitement de choix de l'insuffisance rénale terminale. Outre l'adéquation des antigènes human leucocyte antigens (HLA) entre le donneur et le receveur, la qualité du rein avant la transplantation influence également sa survie. Cette qualité est définie par différents éléments, y compris la sévérité du dommage ischémique lié à la transplantation per se. Le préconditionnement ischémique (PCI) représente un ensemble de manœuvres permettant d'atténuer les lésions d'ischémie/reperfusion (I/R) causées par l'interruption et la reprise de la perfusion rénale, observée notamment lors d'une greffe. Différents modèles animaux du PCI rénal montrent des résultats prometteurs quant à la préservation structurelle et fonctionnelle de l'organe. En outre, la caractérisation des cascades cellulaires d'une I/R a permis d'identifier certains acteurs potentiels du PCI rénal, telles que l'adenosine monophosphate-activated protein kinase (AMPK). L'AMPK est un senseur énergétique impliqué dans le maintien de la polarisation épithéliale en conditions de privation énergétique. L'AMPK est notamment activée par la metformine, classiquement utilisée en diabétologie. Nous résumons ici les données obtenues in vitro et in vivo à propos du rôle de l'AMPK dans le PCI rénal. Définir les conditions d'un PCI pharmacologique permettrait d'optimaliser la qualité d'un greffon rénal avant sa transplantation et prolongerait ainsi sa survie à long terme.

(c) 2013 Association Société de néphrologie. Publié par Elsevier Masson SAS. Tous droits réservés.
\end{abstract}

A B S T R A C T

Kidney transplantation represents the best treatment of end-stage renal disease. In addition to the degree of human leukocyte antigen matching, long-term graft survival is influenced by the quality of the graft before its transplantation. Quality criteria include the level of ischemic damage caused by the transplantation per se. Renal ischemic preconditioning (IP) consists of different approaches to prevent ischemia/reperfusion ( $\mathrm{I} / \mathrm{R}$ ) damage induced by the interruption and recovery of renal circulation, as observed during transplantation. Distinct animal models show promising results regarding the efficiency of PCI to preserve kidney structure and function in I/R conditions. Characterizing the cellular cascades involved in I/R led to the identification of putative targets of renal IP, including the adenosine monophosphate-activated protein kinase (AMPK). AMPK is a ubiquitous energy sensor, which has been implicated in the maintenance of epithelial cell polarization under energy deprivation. Among others, the anti-diabetic drug, metformin, is a potent activator of AMPK. Here, we summarize the in vitro and in vivo data about the role of AMPK in renal IP. Defining the pharmacological conditions of IP would help to improve the quality of the renal graft before its transplantation, thereby increasing its long-term survival.

(c) 2013 Association Société de néphrologie. Published by Elsevier Masson SAS. All rights reserved.

\footnotetext{
* Auteur correspondant.

Adresse e-mail : francois.jouret@chu.ulg.ac.be (F. Jouret).
}

\section{Introduction}

La transplantation rénale représente aujourd'hui le traitement de choix de l'insuffisance rénale terminale (IRT). Cette technique 
permet de réduire les complications, notamment cardiovasculaires, de la dialyse chronique, et ainsi d'améliorer la qualité de vie et la survie des patients en IRT. Cependant, le nombre de patients en liste d'attente d'une greffe rénale ne cesse de croître, surpassant d'année en année le nombre de donneurs. La Belgique fait partie du réseau européen EuroTransplant assurant l'allocation optimale d'organes entre l'Allemagne, l'Autriche, la Belgique, la Croatie, le Luxembourg, les Pays-Bas et la Slovénie (www.eurotransplant. org). En 2011, 3633 transplantations rénales ont été réalisées dans la zone EuroTransplant, dont 482 en Belgique. D'après les derniers chiffres disponibles d'EuroTransplant, le taux de survie d'un rein à 1,3 et 5 ans après la greffe au départ d'un donneur décédé est respectivement de 84,78 et $68 \%$. Dans le cas d'un donneur vivant, les taux de survie du greffon à 1,3 et 5 ans sont respectivement de 93, 89 et $82 \%$. Ces chiffres révèlent la possibilité d'améliorer significativement la survie d'un greffon rénal, en particulier dans le chef des donneurs décédés. Le rejet vasculaire aigu et la dysfonction chronique du greffon représentent les causes principales d'une perte de greffon rénal.

Le degré d'adéquation des antigènes human leucocyte antigens (HLA) entre le donneur et le receveur influence significativement la survie du greffon, en particulier lorsque les organes sont prélevés post-mortem. Cependant, il est curieux de remarquer que le taux de survie du greffon rénal entre les " meilleures " et les " pires " paires de donneur/receveur en termes de compatibilité HLA ne diffère que de l'ordre de $10 \%$ en faveur des paires les plus adéquates (www.eurotransplant.org). Ces observations suggèrent que d'autres facteurs non immunologiques sont impliqués dans la survie à long terme du greffon rénal. Parmi ceux-ci, la qualité de l'organe avant la transplantation joue un rôle critique et peut être définie par différents éléments, tels que :

- l'âge et l'état de santé du donneur ;

- les conditions de prélèvement ;

- les modalités de préservation ;

- les lésions d'ischémie/reperfusion (I/R) liées à la transplantation per se.

La sévérité de l'ischémie rénale diffère selon le type de dons. Lors d'un don vivant, l'ischémie est limitée à une courte période dans le milieu de préservation froide à $4{ }^{\circ} \mathrm{C}$. Un don à cœur battant engendre une plus longue ischémie froide, tandis qu'un don à cœur non battant associe à la fois une ischémie chaude causée par l'arrêt cardiaque et une ischémie froide lors du prélèvement. Tout type de don s'accompagne également d'une phase de reperfusion au moment de la transplantation, qui accentue davantage les lésions induites durant l'ischémie. Par ailleurs, une nouvelle catégorie de donneurs a été récemment créée afin d'augmenter le pool d'organes disponibles : les donneurs à critères étendus. Ces donneurs présentent, par définition, des facteurs de morbidité accrue, tels qu'une hypertension artérielle ou un âge supérieur à 60 ans. Cette co-morbidité augmente la sensibilité du greffon aux phénomènes $\mathrm{d}^{\prime} \mathrm{I} / \mathrm{R}$ [1]. La recherche dans le domaine de la transplantation rénale se focalise donc sur ces différents aspects afin d'améliorer les conditions de prélèvement, de préservation, d'allocation et de transplantation d'un greffon rénal, et partant d'en allonger la survie [1,2]. In fine, ces travaux visent à préparer l'organe avant sa transplantation et ainsi atténuer les lésions $d$ 'I/R. L'ensemble de ces manœuvres se regroupe sous le terme de " préconditionnement ischémique » $(\mathrm{PCI})$.

À l'origine, le PCI a été décrit dans un modèle d'infarctus chez le chien [3]. Son principe est d'exposer l'organe à de brefs épisodes, de l'ordre de 3 à 5 minutes, d'ischémie avant une ischémie prolongée afin de réduire le dommage ischémique et d'accélérer la récupération fonctionnelle. Par la suite, différents modèles expérimentaux utilisant d'autres organes, tels que l'intestin, le cerveau, le foie ou les reins, ont montré que le PCI met les tissus en mode d'économie d'énergie, ce qui préserve leur métabolisme énergétique et réduit l'inflammation lors de la reperfusion [4]. Audelà du PCI chirurgical réalisé par clampages artériels itératifs, de nouvelles approches pharmacologiques se développent en ciblant les différents acteurs de la cascade de l'I/R.

Au niveau cellulaire, l'équilibre énergétique est assuré par une protéine appelée adenosine monophosphate-activated protein kinase (AMPK). De fait, l'AMPK est considérée comme un senseur énergétique capable d'intégrer et d'interpréter le statut énergétique de la cellule, et partant de l'organisme entier $[5,6]$. La consommation excessive d'ATP, forme principale de stockage d'énergie intracellulaire, et l'augmentation subséquente des rapports ADP/ATP ou AMP/ATP entraîne l'activation de l'AMPK. Une cascade synergique d'activation des voies cataboliques produisant de l'énergie et d'inhibition des voies anaboliques consommant de l'ATP est alors enclenchée (Fig. 1).

Compte tenu de l'impact majeur d'une $I / R$ sur l'équilibre énergétique cellulaire et tissulaire, différentes équipes de recherche se sont intéressées au rôle de l'AMPK dans la cascade de l'I/R, et partant dans le PCI rénal. Cet article de revue rappelle la physiopathologie de l'I/R rénale et le concept du PCI avant de détailler l'AMPK ainsi que son rôle potentiel dans le PCI d'un greffon rénal.

\section{Physiopathologie de l'ischémie rénale}

Le prélèvement et la transplantation d'un organe nécessitent l'interruption brutale, mais souvent prévisible, de sa perfusion sanguine. Le débit sanguin rénal au repos est de $1200 \mathrm{~mL} / \mathrm{min}$, soit 20 \% du débit cardiaque, alors que les deux reins représentent moins de $1 \%$ du poids corporel total. En conditions physiologiques, le débit sanguin rénal reste constant jusqu'à une pression artérielle systolique de $80 \mathrm{mmHg}$ grâce à la régulation fine du système nerveux sympathique, de l'axe hormonal rénine-angiotensine et des prostaglandines. L'interruption prolongée de la perfusion sanguine rénale entraîne l'arrêt des apports en nutriments et une chute de la pression partielle en oxygène dans l'organe, provoquant une cascade d'événements cellulaires et tissulaires regroupés sous le vocable de "dommage ischémique " [7,8]. Dans le cadre de la transplantation rénale, le prélèvement de l'organe chez le donneur nécessite l'interruption temporaire et variable (de quelques heures à 36 heures) de la circulation sanguine rénale. Les recommandations actuelles de conservation du greffon rénal prévoient l'utilisation d'une solution physiologique froide afin de ralentir le métabolisme des cellules tubulaires rénales et ainsi limiter le dommage ischémique. Cependant, différents travaux expérimentaux ont montré que les dommages associés à l'hypoperfusion rénale au moment du prélèvement, à la préservation froide et à la reperfusion chaude lors de la transplantation sont responsables de lésions tissulaires significatives érodant la survie du greffon à long terme [1].

Au niveau cellulaire, l'ischémie entraîne une déplétion rapide des réserves énergétiques cellulaires, stockées sous forme d'adénosine triphosphate (ATP), des cellules tubulaires rénales (Fig. 2). Le segment S3 du tubule proximal rénal localisé au niveau de la médulla externe est considéré comme la partie du néphron la plus vulnérable face à un épisode ischémique du fait de sa faible capacité à produire de l'ATP par glycolyse [9]. La consommation d'ATP est de fait maintenue dans un premier temps grâce à la glycolyse anaérobie pour préserver l'homéostasie cellulaire alors que la production d'ATP par phosphorylation oxydative au niveau mitochondrial est interrompue en l'absence d'oxygène. Cette contribution de la glycolyse anaérobie entraîne cependant l'accumulation intracellulaire de lactate et l'acidification du cytoplasme, qui à leur tour perturbent le fonctionnement cellulaire 


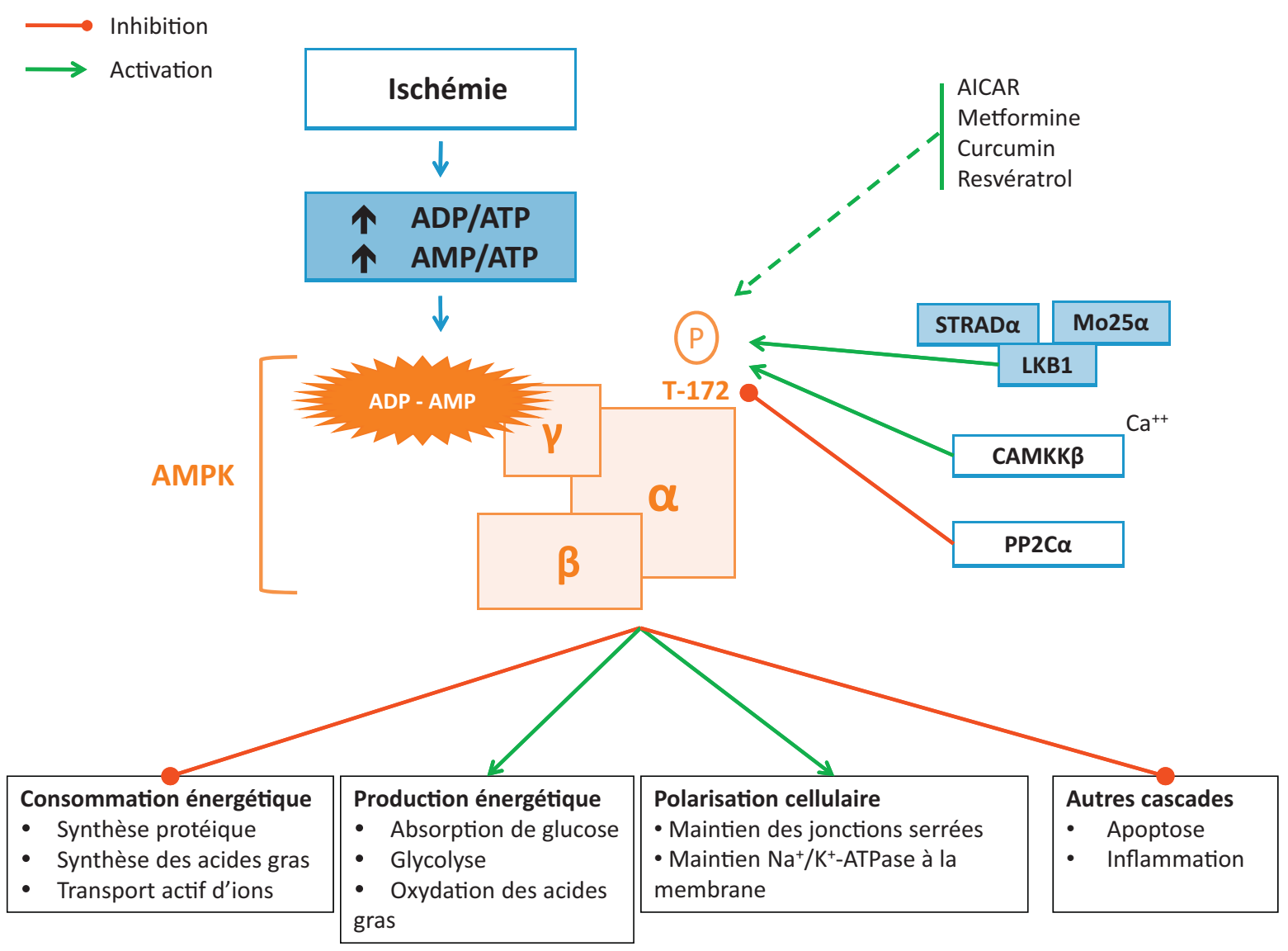

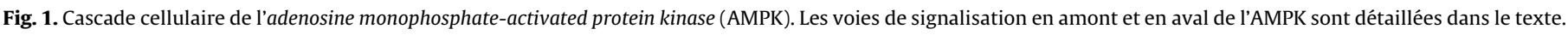

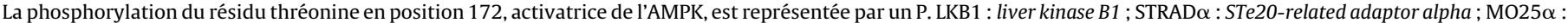

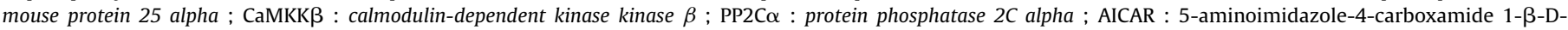
ribofuranoside.

et mitochondrial. Par ailleurs, le manque d'ATP conduit inévitablement à une désorganisation du cytosquelette, un démantèlement des jonctions serrées (JS) intercellulaires, une perte de polarisation épithéliale et un dysfonctionnement de différents transporteurs membranaires, y compris la pompe à sodium, $\mathrm{Na}^{+}$/ $\mathrm{K}^{+}$-ATPase $[7,10]$. L'internalisation de cette pompe entraîne l'accumulation intracellulaire de $\mathrm{Na}^{+}$et un gonflement cellulaire par effet osmotique activant l'échangeur $\mathrm{Na}^{+} / \mathrm{Ca}^{2+}$ responsable d'une augmentation de la concentration cytosolique de $\mathrm{Ca}^{2+}$ $\left[\mathrm{Ca}^{2+}\right]_{\mathrm{i}}$. Cette élévation de $\left[\mathrm{Ca}^{2+}\right]_{i}$ perturbe les cascades enzymatiques dépendantes $\mathrm{du} \mathrm{Ca}^{2+}$ impliquées dans l'organisation du cytosquelette et la mort cellulaire par apoptose [11]. Bien plus, le mauvais adressage membranaire des pompes ioniques empêche la réabsorption ordonnée des solutés et de l'eau de l'ultrafiltrat. Le détachement des cellules épithéliales et endothéliales l'une de l'autre altère respectivement l'étanchéité tubulaire et vasculaire, ce qui, couplé à la diminution de pression interstitielle, provoque un œdème tissulaire majeur. Cet œdème empêchera la bonne reperfusion du greffon rénal au moment de la transplantation. Un cercle vicieux s'installe alors par une boucle de rétrocontrôle tubulo-glomérulaire, induisant la vasoconstriction des artères glomérulaires afférentes et aggravant les lésions ischémiques. La perte cellulaire par nécrose et l'apoptose entraînent la desquamation des cellules épithéliales vers la lumière des tubules rénaux, conduisant à l'obstruction de ces derniers.

Du point de vue métabolomique, le pattern des métabolites endogènes, établi par chromatographie liquide de haute performance couplée à la spectrométrie de masse, apparaît rapidement modifié suite à une $\mathrm{I} / \mathrm{R}$ rénale [12]. Endéans les 2 premières heures suivant une $\mathrm{I} / \mathrm{R}$, la teneur plasmatique et urinaire en lysophospholipides, acides gras libres et nitrotyrosines augmente, tandis que le taux de carnitine et d'acétyl-carnitine diminue significativement. L'activité rénale de la phospholipase A2 augmente également dans le décours d'une $\mathrm{I} / \mathrm{R}$ rénale, à l'inverse de la superoxyde dismutase dont l'activité diminue. Le malondialdéhyde s'accumule du fait de la dégradation oxydative des lipides polyinsaturés, entraînant la formation d'adduits covalents connus sous le nom de " produits de lipoxydation avancée " [13]. En effet, la souffrance des cellules épithéliales lors d'une I/R est associée à la production tissulaire de radicaux libres, sous forme de dérivés oxygénés ou nitrosylés, bien au-delà des capacités antioxydantes cellulaires (Fig. 2). À trop fortes concentrations tissulaires, ces médiateurs chimiques entraînent l'oxydation des composants cellulaires protéiques, lipidiques, membranaires et nucléiques des cellules épithéliales et endothéliales [14,15]. Il est intéressant de noter que le lieu de production accrue de ces radicaux libres correspond aux zones d'apoptose [16]. In vivo, la diminution rapide du taux plasmatique de vitamine E (index de peroxydation lipidique), associée à une augmentation significative du taux plasmatique de myéloperoxydase (marqueur d'une activation des polymorphonucléaires neutrophiles), témoigne d'une production accrue de radicaux libres lors de la phase précoce de reperfusion rénale [17].

L'ensemble de ces conditions physiopathologiques font le lit d'une réaction inflammatoire, caractérisée par l'expression et l'activation des molécules d'adhésion endothéliales et des sélectines. Cette réaction inflammatoire expliquerait en partie pourquoi une ischémie froide plus longue d'un greffon est associée à un 


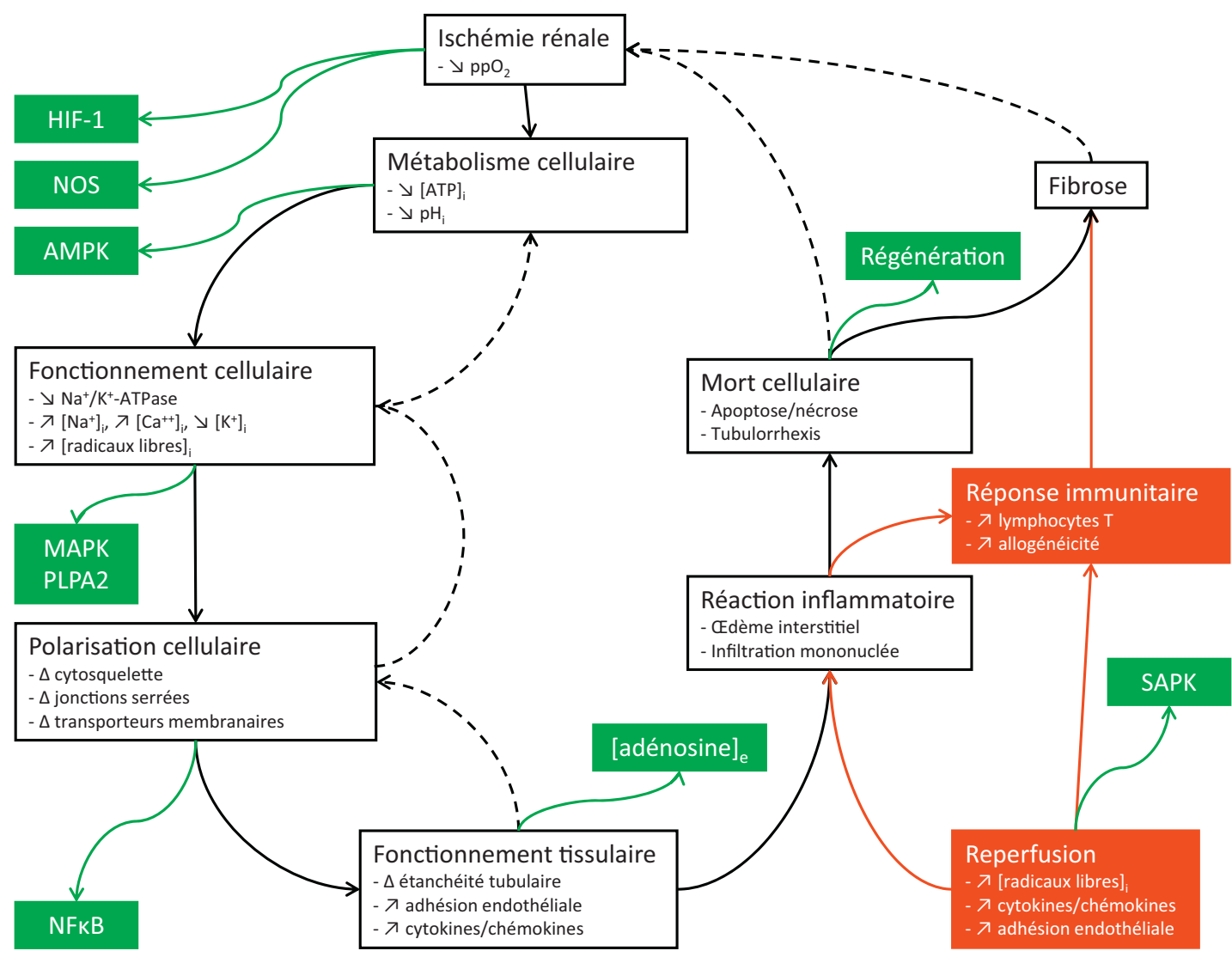

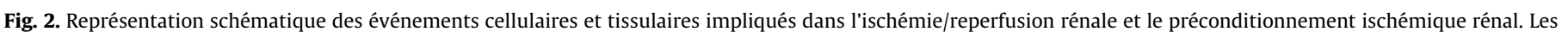

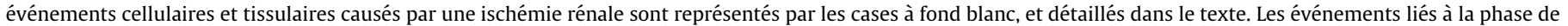

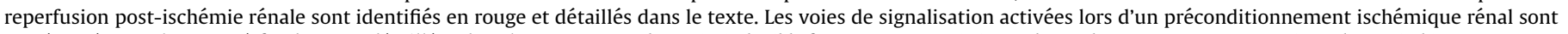

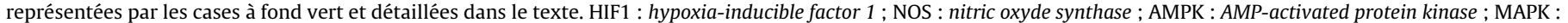
mitogen-activated protein kinases ; PLPA2 : phospholipase A2 ; NFKB : nuclear factor kappa B ; SAPK : stress-activated protein kinases.

risque accru de rejet aigu et/ou de reprise retardée de fonction. Cet effet délétère de l'inflammation consécutive à la reperfusion et l'infiltration de l'organe par les cellules monocytaires est principalement dû à la production localement accrue de radicaux libres et de cytokines/chémokines pro-inflammatoires, telles que le tumor necrosis factor (TNF)- $\alpha$ et l'interleukine (IL)-1 $\beta$, activant différentes kinases liées au stress cellulaire [11]. Enfin, l'ensemble de ces phénomènes liés à l'I/R entraînent des altérations tissulaires irréversibles de type fibrotique, qui à leur tour engendrent et entretiennent une hypoxie chronique de l'organe [18]. Mieux comprendre les mécanismes cellulaires et tissulaires impliqués dans ces phénomènes d'I/R permettrait donc de les exploiter afin de prévenir ou d'atténuer le dommage ischémique, et ainsi accélérer la reprise de fonction du greffon et en augmenter la survie. Il s'agit du principe du PCI rénal.

\section{Principe du PCI rénal et cibles pharmacologiques potentielles}

Le principe du PCI est d'exposer l'organe à de brefs épisodes, de l'ordre de 3 à 5 minutes, d'ischémie avant une conservation en ischémie prolongée afin de réduire le dommage ischémique et accélérer la récupération fonctionnelle [3,4]. Ce stimulus de PCI est efficace lorsqu'il est appliqué directement à l'organe concerné (local ischemic preconditioning) ou à un autre tissu situé à distance de l'organe d'intérêt (remote ischemic preconditioning). Il apparaît donc que des mécanismes de défense sont activés dans certains organes en réponse à un stress énergétique. Plusieurs hypothèses expérimentales sont actuellement en cours d'investigation, à savoir :
- la mise en branle d'une cascade enzymatique de réponses, précoces ou tardives, au stress (stress-related kinases) ;

- la production, locale ou à distance, de cytokines et chémokines modulant l'adhésion des globules blancs à la paroi endothéliale ;

- une dédifférenciation cellulaire responsable d'une plus grande résistance à l'hypoxie et/ou d'une capacité de régénération accélérée (Tableau 1).

In vivo, le PCI rénal permet de maintenir l'organisation du réseau d'actine du cytosquelette et la polarité cellulaire, et d'ainsi préserver la distribution polarisée des transporteurs ioniques, telles que la pompe $\mathrm{Na}^{+} / \mathrm{K}^{+}$-ATPase basolatérale. L'infiltration tissulaire par les leucocytes ainsi que le degré d'apoptose et de nécrose sont significativement moindres lorsqu'un PCI a été préalablement réalisé [19]. Notons une différence de réponse au PCI entre les 2 sexes, à la faveur du sexe féminin [20].

Deux phases principales ont été décrites lors du PCI : la phase précoce (quelques minutes à quelques heures) et la phase tardive (au-delà de 24 heures) (Tableau 1). Les mécanismes cellulaires en jeu lors du PCI sont en cours de caractérisation, et l'importance relative de chacune de ces phases reste controversée [4,8]. Lors de la phase précoce du PCI local, les voies de signalisation du métabolisme énergétique cellulaire et de production de médiateurs chimiques seraient rapidement modulées (Tableau 1). Différentes kinases et phospholipases activées en conditions de stress ont ainsi été incriminées dans le PCI [4]. Le PCI rénal n'a notamment pas d'effet significatif sur l'activation post-ischémique d'extracellular signal-regulated kinase (ERK1/2), mais prévient l'activation des voies Jun N-terminal kinase (JNK) et p38 lors d'un second épisode ischémique [19]. Or, ces kinases participent à 
Tableau 1

Signalisation cellulaire impliquée dans le préconditionnement ischémique.

\begin{tabular}{|c|c|c|}
\hline \multicolumn{3}{|l|}{ Préconditionnement ischémique local } \\
\hline Voies de signalisation & Modèle & Références \\
\hline \multicolumn{3}{|l|}{ Phase précoce } \\
\hline \multirow[t]{2}{*}{ NO synthase } & Rat & $\begin{array}{l}{[5,10,11,13} \\
16,27,35,39]\end{array}$ \\
\hline & Souris & {$[29,38]$} \\
\hline NFKB & Rat & {$[4,6]$} \\
\hline \multirow[t]{3}{*}{ Adénosine } & Souris & [7-9] \\
\hline & Rat & {$[22,33]$} \\
\hline & Chien & {$[24]$} \\
\hline \multirow[t]{3}{*}{ Stress oxydant } & Rat & {$[17,26,34]$} \\
\hline & Lapin & {$[25]$} \\
\hline & Souris & [19] \\
\hline Récepteurs couplés aux protéines G & Rat & [23] \\
\hline Système nerveux autonome & Rat & {$[28,37]$} \\
\hline \multicolumn{3}{|l|}{ Phase tardive } \\
\hline NO synthase & Souris & [16] \\
\hline HSP & Rat & {$[2]$} \\
\hline NFкB & Rat & [14] \\
\hline \multirow[t]{2}{*}{ Stress oxydant } & Rat & [15] \\
\hline & Souris & [18] \\
\hline MAPK & Souris & [30] \\
\hline Cellules circulantes & Souris & {$[12,20,31]$} \\
\hline \multicolumn{3}{|c|}{ Préconditionnement ischémique à distance } \\
\hline Voies de signalisation & Modèle/organe & Références \\
\hline \multicolumn{3}{|l|}{ Phase précoce } \\
\hline TNF $\alpha$ & Rat/foie & {$[1]$} \\
\hline \multirow[t]{2}{*}{ Stress oxydant } & Rat/intestin & [32] \\
\hline & Rat/rein & [34] \\
\hline Adénosine & Rat/membre & {$[36]$} \\
\hline \multicolumn{3}{|l|}{ Phase tardive } \\
\hline \multirow[t]{2}{*}{ Cellules circulantes } & Souris/rein & [3] \\
\hline & Rat/aorte & [21] \\
\hline
\end{tabular}

D’après la méta-analyse de Wever et al. [23]

Les références sont listées dans le Matériel complémentaire.

HSP : heat shock proteins; NFкB : nuclear factor kappa B ; MAPK : mitogen-activated protein kinases ; TNF $\alpha$ : tumor necrosis factor- $\alpha$.

l'expression des molécules d'adhésion endothéliale et à la production de cytokines responsables du recrutement des leucocytes au niveau des petits vaisseaux de la médullaire externe. Leur inhibition causée par un PCI permettrait de prévenir l'infiltration monocytaire et de réduire l'inflammation tissulaire post-ischémique. En outre, le rein préconditionné se caractérise par une activité accrue de l'hème oxygénase et des isoformes inductibles (inducible nitric oxide synthase [iNOS]) et endothéliales (eNOS), ce qui suggère que l'oxyde nitrique (NO) participe au $\mathrm{PCI}$ [21]. La baisse de la pression partielle en oxygène dans le parenchyme rénal entraîne l'activation du facteur de transcription hypoxia-inducible factors (HIF-1), qui à son tour régule différents gènes impliqués dans la survie cellulaire. Enfin, l'adénosine est massivement produite lors de courts épisodes d'ischémie. Ceci protégerait le rein ischémique en inhibant l'adhésion leucocytaire $[4,11]$. Différentes études utilisant des agonistes et des antagonistes et/ou des modèles murins génétiquement modifiés suggèrent que les récepteurs $\mathrm{A} 1$ et $\mathrm{A} 2 \mathrm{a}$ participent au $\mathrm{PCI}$ rénal, tandis que le récepteur $A 3$ a un effet délétère lors d'une $I / R$ via son possible rôle dans l'activation de l'apoptose [22]. Lors de la phase tardive, l'expression des gènes des cascades de l'inflammation, de la dédifférenciation cellulaire et de l'apoptose est finement régulée, notamment par le facteur de transcription nuclear factor kappa $B$ (NFKB) (Tableau 1).

Chez l'animal, plusieurs travaux ont montré que le PCI chirurgical, local ou à distance, est associé à une diminution du dommage ischémique rénal, tel que résumé récemment par la méta-analyse de Wever et al. [23]. Les paramètres mesurés dans cette méta-analyse comprenaient les taux de créatinine sérique et d'urée, ainsi que le score histologique de Jablonski après $I / R$, avec versus sans $\mathrm{PCI}$. Cette étude suggère que la phase tardive du PCI rénal (au-delà de 24 heures) serait plus efficace que la phase précoce. En outre, la masse de tissu utilisé au moment du PCI à distance semble être un critère majeur de son efficacité. Les mécanismes cellulaires impliqués dans le PCI à distance demeurent méconnus et impliqueraient la voie neurogénique autonome et la sécrétion de divers messagers biochimiques dans la circulation sanguine [24]. Une étude récente utilisant un modèle d'ischémie brève et répétée du membre inférieur avant une $I / R$ rénale chez le rat suggère que ce type de $\mathrm{PCI}$ à distance résulterait d'une sécrétion accrue d'endorphines [25]. En effet, un prétraitement des animaux par la naloxone, antagoniste non sélectif des récepteurs opioïdes, abolit l'effet protecteur d'un PCI rénal par ischémies brèves et répétées du membre inférieur, tant du point de vue fonctionnel que structurel. En revanche, l'inhibition pharmacologique d'autres voies de signalisation impliquées dans le PCI à distance cardiaque, telles que la noradrénaline, le système cannabinoïde, les glucocorticoïdes, la NO synthase inductible, le calcitonin gene-related peptide (CGRP), la voie médiée par les ganglions, l'hème oxygénase et les radicaux libres, n'avait pas d'impact significatif sur l'effet bénéfique d'une ischémie brève et répétée du membre inférieur avant une $\mathrm{I} / \mathrm{R}$ rénale. Ces observations confirment que les mécanismes tissulaires et cellulaires impliqués dans le PCI diffèrent d'un organe à un autre selon l'espèce animale et le protocole expérimental [25].

Chez l'homme, il n'existe pas, à ce jour, d'étude sur l'effet du PCI local sur la protection rénale. Quelques études utilisant des protocoles divergents de $\mathrm{PCI}$ à distance, dans des conditions pathologiques autres que la transplantation (la chirurgie cardiaque ou aortique par exemple), ont été réalisées, avec des résultats préliminaires inconstants [26]. Notons que quasiment toutes les études chez l'homme, enregistrées auprès de l'agence Clinicaltrials.gov, s'intéressent à la phase précoce du PCI. Dans le domaine de la transplantation rénale, le PCI pourrait être utilisé au moment du prélèvement de l'organe afin de limiter les lésions des cellules tubulaires rénales et prévenir la mort des cellules endothéliales vasculaires [1,2]. Cependant, la réalisation d'un PCI chirurgical au moment du prélèvement d'un rein risquerait d'endommager les structures vasculaires. Il est dès lors nécessaire d'identifier les cibles cellulaires et tissulaires du PCI rénal, local ou à distance, afin de développer une approche pharmacologique prudente et efficace. Différentes cibles ont été testées à ce jour chez le rongeur et sont listées dans le Tableau 2. Dans cet article de revue, nous détaillons l'une de ces cibles prometteuses, à savoir l'AMPK.

\section{AMP-activated protein kinase (AMPK)}

L'AMPK est une sérine/thréonine kinase ubiquitaire très conservée sur le plan phylogénétique. L'AMPK des mammifères forme un complexe hétérotrimérique regroupant une sous-unité catalytique, alpha, et deux sous-unités régulatrices, bêta et gamma (Fig. 1). Chacune de ces sous-unités existe sous différentes isoformes $(\alpha 2, \beta 2, \gamma 3)$, ce qui permet 12 combinaisons hétérotrimériques possibles. Les gènes codant pour ces différentes sousunités sont identifiables dans d'autres familles d'eucaryotes plus primitifs, telles que les plantes, les champignons et les protistes [5].

La sous-unité $\alpha$ renferme le domaine catalytique de l'AMPK dans sa portion N-terminale et n'est active qu'après phosphorylation de la thréonine en position 172 par une autre kinase. La sousunité $\beta$ possède un domaine de liaison du glycogène, qui lui permettrait de jauger directement les réserves énergétiques de la cellule. La sous-unité $\gamma$ contient 2 sites de liaison antagoniste des nucléotides (Fig. 1). L'augmentation des rapports ADP/ATP ou AMP/ATP, qui signalent un statut énergétique cellulaire faible, 
Tableau 2

Exemples de préconditionnement ischémique rénal pharmacologique chez le rongeur.

\begin{tabular}{|c|c|c|}
\hline Cascades cellulaires & Cibles cellulaires & Médicaments \\
\hline Hypoxie & HIF- $1 \alpha$ & $\begin{array}{l}\text { Érythropoïétine } \\
\text { Isoflurane }\end{array}$ \\
\hline Métabolisme & $\begin{array}{l}\text { AMPK } \\
\text { Hmox1 inducer }\end{array}$ & $\begin{array}{l}\text { AICAR } \\
\text { Metformine } \\
\text { Resvératrol } \\
\text { Hemin }\end{array}$ \\
\hline Stress oxydant & Radicaux libres & $\begin{array}{l}\text { L-carnitine } \\
\text { Danshen } \\
\text { Éthanol } \\
\text { N-acétylcystéine } \\
\text { Lithium } \\
\text { NONOate spermine }\end{array}$ \\
\hline Inflammation & $\begin{array}{l}\text { MAPK } \\
\text { RCPG }\end{array}$ & $\begin{array}{l}\text { Cyclosporine } \\
\text { FK-506 } \\
\text { Adénosine (et agonistes) } \\
\text { Apyrase (CD39 soluble) } \\
\text { Catécholamines } \\
\text { Cellules souches } \\
\text { mésenchymateuses }\end{array}$ \\
\hline Réparation & & $\begin{array}{l}\text { Cellules souches } \\
\text { mésenchymateuses }\end{array}$ \\
\hline
\end{tabular}

$\overline{\text { HIF-1 : hypoxia-inducible factor } 1 \text {; AMPK : AMP-activated protein kinase ; NOS : nitric }}$ oxyde synthase ; MAPK : mitogen-activated protein kinases ; RCPG : récepteurs couplés aux protéines $\mathrm{G}$.

entraîne la liaison d'ADP ou d'AMP à la sous-unité $\gamma$. Cet événement permet la phosphorylation et l'activation de la sousunité $\alpha$, et s'accompagne de modifications allostériques de l'AMPK phosphorylée, la rendant résistante aux phosphatases et amplifiant davantage son activité [5]. La principale kinase responsable de cette phosphorylation activatrice est la liver kinase B1 (LKB1). LKB1 fait partie de la famille des suppresseurs de tumeur et interagit avec deux partenaires principaux, STRAD $\alpha$ et MO25 $\alpha$. Une autre kinase également capable de phosphoryler et d'activer l'AMPK est la calmodulin-dependent kinase kinase $\beta$ (СаMKK $\beta$ ). Cette kinase est sensible aux variations de la concentration cytosolique en $\mathrm{Ca}^{2+}$, mais pas aux variations du rapport AMP/ATP. La contribution relative de LKB1, CaMKK $\beta$ ou d'autres AMPK kinases dans les cellules tubulaires rénales reste méconnue [27]. Il existe enfin des activateurs pharmacologiques d'AMPK, comme la metformine et l'AICAR (5-aminoimidazole-4-carboxamide $1-\beta-D-$ ribofuranoside), dont le mécanisme d'action reste débattu (Fig. 1).

L'AMPK est considérée comme un senseur énergétique capable d'intégrer et d'interpréter le statut énergétique systémique et cellulaire [5,6]. La consommation excessive d'ATP, forme principale de stockage d'énergie intracellulaire, entraîne l'activation de l'AMPK. Une cascade synergique d'activation des voies cataboliques produisant de l'énergie et d'inhibition des voies anaboliques consommant de l'ATP est alors enclenchée (Fig. 1). Ainsi, l'AMPK activée freine la synthèse des macromolécules, telles que les protéines, les acides gras, les lipides, le cholestérol et le glycogène, et active la $\beta$-oxydation des acides gras, l'absorption du glucose et la glycolyse afin de restaurer l'équilibre énergétique [6]. Grâce aux modèles murins génétiquement invalidés pour les différentes isoformes de la sous-unité catalytique $\alpha$ de l'AMPK, son rôle dans l'équilibre métabolique a pu être mieux caractérisé. À l'échelle de l'organisme entier, les complexes AMPK incluant l'isoforme $\alpha 2$ participeraient au rétrocontrôle des signaux métaboliques périphériques via le système nerveux autonome orthosympathique [28]. Au niveau rénal, l'isoforme $\alpha 1$ prédomine et participe à la régulation énergétique des cellules tubulaires et au couplage métabolisme/transport ionique [27]. Ainsi, l'exposition des cellules tubulaires à l'activateur de l'AMPK, AICAR, induit la translocation membranaire du glucose transporter 1 (transporteur GLUT1) permettant l'entrée du glucose, principal substrat énergétique. À l'inverse, l'activation d'AMPK s'accompagne de la phosphorylation et l'inhibition de différents transporteurs membranaires du $\mathrm{Na}^{+}$, du $\mathrm{K}^{+}$ou du $\mathrm{Cl}^{-}$[27]. De façon intéressante, l'impact d'AMPK sur la distribution et l'activité de la pompe $\mathrm{Na}^{+} /$ $\mathrm{K}^{+}$-ATPase, moteur majeur du gradient ionique transmembranaire, varie d'un type cellulaire à un autre. En effet, l'activation d'AMPK dans les cellules épithéliales alvéolaires H441 ou A549 lors d'une hypoxie ou d'un traitement pharmacologique entraîne l'inhibition de cette pompe [29,30]. En revanche, l'activation de l'AMPK dans les cellules rénales Marti-Darby canine kidney (MDCK) promeut l'expression et le maintien de cette pompe à la membrane basolatérale en conditions physiologiques et de stress énergétique [10,31]. Ces observations suggèrent que les partenaires de l'AMPK changent selon le type cellulaire et selon les conditions expérimentales.

Outre son rôle de senseur énergétique, l'AMPK participe à d'autres fonctions cellulaires, y compris l'inflammation, la prolifération et la différenciation cellulaire, ainsi que la formation et le maintien des JS des cellules épithéliales. Les épithelia constituent la limite physiologique entre un organisme et son environnement. Leur capacité à assurer un transport vectoriel de fluide et de solutés est indispensable à l'homéostasie de cet organisme [32]. Afin d'assumer cette fonction, les cellules épithéliales polarisent leur membrane plasmique en domaines morphologiquement et fonctionnellement distincts, séparés par les JS. Les JS forment ainsi un ciment intercellulaire dynamique qui contrôle la perméabilité paracellulaire et participe à divers processus intracellulaires. L'organisation complexe et dynamique des JS requiert de l'énergie. Par conséquent, tout changement significatif du métabolisme cellulaire, tel une ischémie, s'accompagne de la dislocation des JS et la dépolarisation des transporteurs membranaires [7]. Certains mécanismes de contrôle du métabolisme cellulaire, y compris l'AMPK, sont ainsi impliqués dans la régulation des JS. Dans le modèle in vitro des cellules rénales MDCK, l'assemblage des JS est facilité lors de l'activation pharmacologique de l'AMPK par la metformine ou l'AICAR, et freiné en l'absence d'AMPK [10,33,34]. Au contraire, la dislocation des JS induite par l'exposition des cellules MDCK à une privation énergétique ou à un milieu extracellulaire pauvre en $\mathrm{Ca}^{2+}$ est ralentie si l'AMPK est simultanément activée par le composé AICAR $[10,34]$. In vivo, le démantèlement des JS et la perte de polarisation des cellules tubulaires représentent l'une des premières manifestations histologiques de souffrance ischémique rénale [7]. Vu que l'AMPK est abondamment exprimée dans différents segments du néphron, il était tentant de postuler qu'une privation énergétique rénale, telle que celle observée lors d'une ischémie, s'accompagnerait d'une activation de l'AMPK, et que l'activation pharmacologique de l'AMPK avant un épisode d'ischémie/reperfusion maintiendrait la distribution polarisée des transporteurs membranaires dans les cellules tubulaires rénales, atténuerait le dommage ischémique, et participerait ainsi aux mécanismes de protection rénale [2].

\section{AMPK et préconditionnement ischémique rénal}

En cardiologie, le rôle de l'AMPK a été étudié dans différents modèles ex vivo et in vivo d'infarctus myocardique. L'utilisation d'un modèle murin transgénique exprimant une isoforme non fonctionnelle de l'AMPK $\alpha 2$, sous une forme dominante négative (mutation K45R) dans les cellules musculaires cardiaques et squelettiques, montre que la récupération cardiaque fonctionnelle de ces animaux après un infarctus myocardique est moindre que chez les souris contrôles [35]. À l'inverse, la stimulation directe ex vivo de l'AMPK par le composé A-769662 diminue la nécrose 
myocardique et améliore la récupération de la contractilité du ventricule gauche après un épisode d'I/R par rapport à des cœurs non traités [36]. Ceci s'accompagne d'une activation de la forme endothéliale de la nitric oxide synthase (eNOS) au moment de l'ischémie, ainsi que d'une translocation membranaire du transporteur de glucose, GLUT4. Les cœurs des souris invalidés pour le gène de l'AMPK sont insensibles à l'A-769662, ce qui établit la spécificité du composé pharmacologique. Notons cependant que les cœurs de ces animaux déficients en AMPK $\alpha 2$ répondent au $\mathrm{PCI}$ de façon similaire aux souris contrôles [36]. Dans le poumon, la préactivation pharmacologique de l'AMPK par AICAR est également associée à une diminution significative des lésions induites par l'endotoxine lipopolysaccharide [37].

En 2005, l'équipe de Mount et al. a montré, dans un modèle d'ischémie rénale chez le rat Sprague-Dawley, que l'AMPK est phosphorylée et activée dès le clampage du pédicule vasculaire rénal, en particulier au niveau des tubules corticaux [38]. Cependant, contrairement aux observations faites dans le cœur, l'activation de l'AMPK durant l'ischémie rénale ne s'accompagne pas d'une phosphorylation de l'enzyme eNOS. Les cellules rénales sont par ailleurs dépourvues de GLUT4. Les cibles de l'AMPK durant le PCI des cellules épithéliales rénales demeurent méconnues et diffèrent manifestement de celles identifiées dans les cardiomyocytes [1]. De façon similaire, une étude récente utilisant un modèle d'ischémie brève et répétée du membre inférieur avant une $I / R$ rénale chez le rat a démontré que les mécanismes tissulaires et cellulaires impliqués dans le PCI diffèrent d'un organe à un autre, selon l'espèce animale et le protocole expérimental [25]. Plus récemment, deux groupes indépendants ont suggéré que la préactivation pharmacologique de l'AMPK in vivo par metformine ou par AICAR protège les reins des dommages causés par un épisode d'I/R $[10,39]$. Les travaux de Seo-Mayer et al. montrent, dans un modèle d'ischémie (30 minutes)/reperfusion (48 heures) chez la souris, que l'administration intrapéritonéale de metformine ( $300 \mathrm{mg} / \mathrm{kg}$ par jour) pendant 3 jours avant clampage des pédicules vasculaires rénaux préserve l'architecture des cellules tubulaires et la distribution membranaire de la pompe $\mathrm{Na}^{+} / \mathrm{K}^{+}$-ATPase. D'un point de vue métabolique, ce maintien d'un transporteur énergivore à la surface des cellules épithéliales apparaît contreintuitif. En effet, la stimulation de l'AMPK responsable d'un switch cellulaire vers un mode " économie d'énergie " devrait s'accompagner d'une internalisation et/ou d'une inhibition de cette pompe, comme cela est observé dans des cellules alvéolaires [29,30]. L'explication des auteurs s'appuie sur les conséquences physiologiques dramatiques qu'engendrerait l'internalisation de la pompe $\mathrm{Na}^{+} / \mathrm{K}^{+}$-ATPase dans les cellules rénales, à savoir une perte des capacités de réabsorption tubulaire de l'eau et des solutés, une diurèse massive, une déplétion volémique et une perturbation sévère de l'organisme [10]. La pompe $\mathrm{Na}^{+} / \mathrm{K}^{+}$-ATPase ne serait dès lors pas une cible énergétique lors d'un PCI rénal [3]. Les taux sériques de créatinine et d'urée après $\mathrm{I} / \mathrm{R}$ rénale étaient similaires entre le groupe témoin et le groupe prétraité par la metformine, mais l'excrétion urinaire du biomarqueur de souffrance rénale, neutrophil gelatinase-associated lipocalin (NGAL), était significativement réduite chez les souris traitées par metformine avant l'I/R. Chez le rat, les observations de Lempiäinen et al. démontrent que l'administration intraveineuse de doses croissantes d'AICAR (50 à $500 \mathrm{mg} / \mathrm{kg}$ ), 1 heure avant clampage des pédicules vasculaires rénaux, durant 40 minutes, prévient les lésions de nécrose tubulaire aiguë à 24 heures, atténue l'infiltration monocytaire et le stress oxydatif, et diminue les taux sériques de créatinine et d'urée [39]. Notons cependant que l'absence d'AMPK dans un modèle murin génétiquement invalidé pour la sous-unité $\beta_{1}{ }^{-1-}$ n'aggrave pas les lésions histologiques causées par un épisode d'I/R [40]. Enfin, les travaux de Lin et al. portant sur un modèle canin de transplantation rénale autologue ont montré qu'un traitement combiné par AICAR et l'antioxydant, N-acétylcystéine, atténue les lésions d'I/R rénales [41].

L'ensemble de ces observations supporte que la préactivation pharmacologique de l'AMPK avant un épisode d'I/R atténue le dommage ischémique des cellules tubulaires rénales. Plusieurs facteurs pourraient expliquer ce rôle protecteur (Fig. 1). La préactivation d'AMPK pourrait inhiber les transporteurs ioniques des cellules tubulaires rénales [27] et ainsi réduire la consommation d'énergie dans le contexte métaboliquement vulnérable qu'est l'ischémie. En outre, l'activation d'AMPK a été associée à un état anti-inflammatoire incluant la diminution des voies du TNF $\alpha$, de la NO synthase inductible et de l'interleukine-6 [42]. Enfin, l'activation de l'AMPK faciliterait l'action des protéines heat shock proteins (HSP) au niveau rénal. Ces protéines HSP sont des chaperons moléculaires intervenant dans la préservation et la restitution de la polarité épithéliale lors d'une I/R rénale, notamment par stabilisation de la pompe $\mathrm{Na}^{+} / \mathrm{K}^{+}$-ATPase [43]. Les mécanismes cellulaires et tissulaires en jeu, et en particulier l'impact de l'AMPK sur le transport transépithélial du $\mathrm{Na}^{+}$et des autres solutés au moment de l'ischémie et durant la phase de reperfusion, sont en cours de caractérisation. Il serait également utile d'investiguer le rôle de l'AMPK au cours des phases d'inflammation et de régénération cellulaire post-I/R rénale.

\section{Conclusions et perspectives}

La transplantation rénale représente aujourd'hui le traitement de choix de l'IRT. Dans le monde entier, l'inadéquation grandissante entre le nombre de patients en attente d'un greffon et le nombre d'organes disponibles motive l'assouplissement des critères d'éligibilité des donneurs. La qualité du rein avant la transplantation conditionne cependant sa sensibilité au dommage ischémique, et partant influence sa survie à long terme. Les modèles animaux du PCI rénal montrent des résultats prometteurs quant à la préservation structurelle et fonctionnelle de l'organe. Les études actuellement disponibles chez l'homme sont rares et préliminaires. Une meilleure caractérisation des cascades cellulaires et tissulaires en jeu lors des phénomènes $d$ 'I/R permettra d'identifier de nouvelles cibles pharmacologiques du PCI rénal, telles que l'AMPK. L'activation directe de l'AMPK par différents composés prévient de fait le dommage ischémique chez l'animal. Son rôle dans le PCI rénal chez l'homme est inconnu. De même, l'identification des mécanismes en amont et en aval de l'AMPK dans le PCI rénal est un champ d'investigations nouveau dans le domaine de la transplantation. Optimaliser les conditions de prélèvement et de conservation améliorerait la qualité du greffon rénal avant sa transplantation et prolongerait ainsi sa survie à long terme.

\section{Déclaration d'intérêts}

Les auteurs déclarent ne pas avoir de conflits d'intérêts en relation avec cet article.

\section{Remerciements}

Les auteurs remercient l'équipe chirurgicale ( $M$. Meurisse, C. Coimbra Marques, A. De Roover, O. Detry, E. Hamoir, P. Honoré, L. Kohnen, J.-P. Squifflet) et médicale (C. Bonvoisin, S. Grosch, L. Weekers et P. Xhignesse) de transplantation, ainsi que les coordinatrices de greffe (M.-H. Delbouille, M.-H. Hans, J. Mornard), pour la qualité de leur investissement personnel et professionnel dans la transplantation rénale au centre hospitalier universitaire de Liège. François Jouret a un mandat de clinicien spécialiste post-doctorant du Fonds national de la recherche scientifique au sein de l'unité GIGA Cardiovascular 
Sciences (J.-O. Defraigne et J.-M. Krzesinski). Ce projet est soutenu par une subvention de l'Association du Centre d'information sur l'insuffisance rénale et ses traitements dans le cadre du Fonds Léon-Frédéricq.

\section{Annexe A. Matériel complémentaire}

Le matériel complémentaire accompagnant la version en ligne de cet article est disponible sur http://www.sciencedirect.com et http://dx.doi.org/10.1016/j.nephro.2013.10.006.

\section{Références}

[1] Bon D, Chatauret N, Giraud S, Thuillier R, Favreau F, Hauet T. New strategies to optimize kidney recovery and preservation in transplantation. Nat Rev Nephrol 2012;8:339-47.

[2] Bouma HR, Ketelaar ME, Yard BA, Ploeg RJ, Henning RH. AMP-activated protein kinase as a target for preconditioning in transplantation medicine. Transplantation 2010;90:353-8.

[3] Murry CE, Jennings RB, Reimer KA. Preconditioning with ischemia: a delay of lethal cell injury in ischemic myocardium. Circulation 1986;74:1124-36.

[4] Bonventre JV. Kidney ischemic preconditioning. Curr Opin Nephrol Hypertens $2002 ; 11: 43-8$

[5] Hardie DG, Ross FA, Hawley SA. AMPK: a nutrient and energy sensor that maintains energy homeostasis. Nat Rev Mol Cell Biol 2012;13:251-62.

[6] Viollet B, Athea Y, Mounier R, Guigas B, Zarrinpashneh E, Horman S, et al. AMPK: lessons from transgenic and knockout animals. Front Biosci 2009;14: 19-44.

[7] Schrier RW, Wang W, Poole B, Mitra A. Acute renal failure: definitions, diagnosis, pathogenesis, and therapy. J Clin Invest 2004;114:5-14.

[8] Bouma HR, Ploeg RJ, Schuurs TA. Signal transduction pathways involved in brain death-induced renal injury. Am J Transplant 2009;9:989.

[9] Bagnasco S, Good D, Balaban R, Burg M. Lactate production in isolated segments of the rat nephron. Am J Physiol 1985;248:F522-6.

[10] Seo-Mayer PW, Thulin G, Zhang L, Alves DS, Ardito T, Kashgarian M, et al. Preactivation of AMPK by metformin may ameliorate the epithelial cell damage caused by renal ischemia. Am J Physiol Renal Physiol 2011;301: F1346-57.

[11] Favreau F, Giraud S, Bon D, Chatauret N, Thuillier R, Hauet T. Ischemia reperfusion control: the key of kidney graft outcome. Med Sci (Paris) 2013;29:183-8

[12] Liu Y, Yan S, Ji C, Dai W, Hu W, Zhang W, et al. Metabolomic changes and protective effect of (L)-carnitine in rat kidney ischemia/reperfusion injury. Kidney Blood Press Res 2012;35:373-81.

[13] Vlassara H. Advanced glycation in health and disease: role of the modern environment. Ann N Y Acad Sci 2005;1043:452-60.

[14] Li C, Jackson RM. Reactive species mechanisms of cellular hypoxia-reoxygenation injury. Am J Physiol Cell Physiol 2002;282:C227-41.

[15] Defraigne JO, Pincemail J, Franssen C, Meurisse M, Defechereux T, Philippart C, et al. In vivo free radical production after cross-clamping and reperfusion of the renal artery in the rabbit. Cardiovasc Surg 1993;1:343-9.

[16] Chien CT, Lee PH, Chen CF, Ma MC, Lai MK, Hsu SM. De novo demonstration and co-localization of free-radical production and apoptosis formation in rat kidney subjected to ischemia/reperfusion. J Am Soc Nephrol 2001;12:973-82.

[17] Pincemail J, Defraigne JO, Franssen C, Bonnet P, Deby-Dupont G, Pirenne J, et al. Evidence for free radical formation during human kidney transplantation. Free Radic Biol Med 1993;15:343-8.

[18] Wang W, Koka V, Lan HY. Transforming growth factor-beta and Smad signalling in kidney diseases. Nephrology 2005;10:48-56.

[19] Park KM, Chen A, Bonventre JV. Prevention of kidney ischemia/reperfusioninduced functional injury and JNK, p38, and MAPK kinase activation by remote ischemic pretreatment. J Biol Chem 2001;276:11870-6.

[20] Pitcher JM, Wang M, Tsai BM, Kher A, Turrentine MW, Brown JW, et al. Preconditioning: gender effects. J Surg Res 2005;129:202-20.

[21] Park KM, Byun JY, Kramers C, Kim JI, Huang PL, Bonventre JV. Inducible nitricoxide synthase is an important contributor to prolonged protective effects of ischemic preconditioning in the mouse kidney. J Biol Chem 2003;278:27256-6.
[22] Soullier S, Gayrard N, Méjean C, Swarcz I, Mourad G, Argilés A. Molecular mechanisms involved in kidney ischemia-reperfusion. Nephrol Ther 2005;1:315-21.

[23] Wever KE, Menting TP, Rovers M, van der Vliet JA, Rongen GA, Masereeuw R, et al. Ischemic preconditioning in the animal kidney, a systematic review and meta-analysis. PLoS One 2012;7:e32296.

[24] Tapuria N, Kumar Y, Habib MM. Remote ischemic preconditioning: a novel protective method from ischemia reperfusion injury - a review. J Surg Res 2008;2:304-30.

[25] Wever KE, Masereeuw R, Wagener FA, Verweij VG, Peters JG, Pertijs JC, et al. Humoral signalling compounds in remote ischaemic preconditioning of the kidney, a role for the opioid receptor. Nephrol Dial Transplant 2013;28: 1721-32.

[26] Hausenloy DJ, Yellon DM. The therapeutic potential of ischemic conditioning: an update. Nat Rev Cardiol 2011;8:619-29.

[27] Pastor-Soler NM, Hallows KR. AMP-activated protein kinase regulation of kidney tubular transport. Curr Opin Nephrol Hypertens 2012;21:523-33.

[28] Viollet B, Andreelli F, Jørgensen SB, Perrin C, Geloen A, Flamez D, et al. The AMP-activated protein kinase alpha2 catalytic subunit controls whole-body insulin sensitivity. J Clin Invest 2003;111:91-8.

[29] Gusarova GA, Trejo HE, Dada LA, Briva A, Welch LC, Hamanaka RB, et al Hypoxia leads to Na,K-ATPase downregulation via $\mathrm{Ca} 2 \mathrm{~b}$ release-activated Ca2b channels and AMPK activation. Mol Cell Biol 2011:31:3546-56.

[30] Tan CD, Smolenski RT, Harhun MI, Patel HK, Ahmed SG, Wanisch K, et al. AMPactivated protein kinase (AMPK)-dependent and -independent pathways regulate hypoxic inhibition of transepithelial $\mathrm{Na}+$ transport across human airway epithelial cells. Br J Pharmacol 2012;167:368-82.

[31] Alves DS, Farr GA, Seo-Mayer P, Caplan MJ. AS160 associates with the $\mathrm{Na}^{+}, \mathrm{K}^{+}-$ ATPase and mediates the adenosine monophosphate-stimulated protein kinase-dependent regulation of sodium pump surface expression. Mol Biol Cell 2010;21:4400-8

[32] Caplan MJ, Seo-Mayer P, Zhang L. Epithelial junctions and polarity: complexes and kinases. Curr Opin Nephrol Hypertens 2008;17:506-12.

[33] Zhang L, Li J, Young LH, Caplan MJ. AMP-activated protein kinase regulates the assembly of epithelial tight junctions. Proc Natl Acad Sci U S A 2006;103: 17272-7.

[34] Zhang L, Jouret F, Rinehart J, Sfakianos J, Mellman I, Lifton RP, et al. AMPactivated protein kinase (AMPK) activation and glycogen synthase kinase$3 \beta$ (GSK-3 $\beta$ ) inhibition induce Ca2+-independent deposition of tight junction components at the plasma membrane. J Biol Chem 2011;286: 16879-90.

[35] Russell 3rd RR, Li J, Coven D, Pypaert M, Zechner C, Palmeri M, et al. AMPactivated protein kinase mediates ischemic glucose uptake and prevents postischemic cardiac dysfunction, apoptosis, and injury. J Clin Invest 2004;114: 495-503.

[36] Kim AS, Miller EJ, Wright TM, Li J, Qi D, Atsina K, et al. A small molecule AMPK activator protects the heart against ischemia-reperfusion injury. J Mol Cell Cardiol 2011:51:24-32.

[37] Zhao X, Zmijewski J, Lorne E, Liu G, Park Y, Tsuruta Y, et al. Activation of AMPK attenuates neutrophil proinflammatory activity and decreases the severity of acute lung injury. Am J Physiol Lung Cell Mol Physiol 2008;295:L497-504.

[38] Mount PF, Hill RE, Fraser SA, Levidiotis V, Katsis F, Kemp BE, et al. Acute renal ischemia rapidly activates the energy sensor AMPK but does not increase phosphorylation of eNOS-Ser1177. Am J Physiol Renal Physiol 2005;289: F1103-15.

[39] Lempiäinen J, Finckenberg P, Levijoki J, Mervaala E. AMPK activator AICAR ameliorates ischaemia reperfusion injury in the rat kidney. $\mathrm{Br} \mathrm{J}$ Pharmacol 2012:166:1905-15.

[40] Mount PF, Gleich K, Tam S, Fraser SA, Choy SW, Dwyer KM, et al. The outcome of renal ischemia-reperfusion injury is unchanged in AMPK- $\beta 1$ deficient mice. PLoS One 2012;7:e29887.

[41] Lin A, Sekhon C, Sekhon B, Smith A, Chavin K, Orak J, et al. Attenuation of ischemia-reperfusion injury in a canine model of autologous renal transplantation. Transplantation 2004;78:654-9.

[42] Peairs A, Radjavi A, Davis S, Li L, Ahmed A, Giri S, et al. Activation of AMPK inhibits inflammation in MRL/lpr mouse mesangial cells. Clin Exp Immunol 2009; 156:542-51.

[43] Riordan M, Sreedharan R, Wang S, Thulin G, Mann A, Stankewich M, et al. HSP70 binding modulates detachment of Na-K-ATPase following energy deprivation in renal epithelial cells. Am J Physiol Renal Physiol 2005;288: F1236-42. 